\title{
The Roles of Oncogenes and Tumor Supressor Genes in Breast Cancer Pathogenesis
}

\author{
Havva Hande Keser Şahin ${ }^{1}$, Mustafa Şahin ${ }^{2}$ \\ ${ }^{1}$ Hitit University Faculty of Medicine, Department of Medical Pathology, Çorum, Turkey \\ ${ }^{2}$ Hitit University Erol Olçok Training and Research Hospital, Department of Medical Biochemistry, Çorum, \\ Turkey
}

*Corresponding Author: Dr. Havva Hande Keser Şahin, Hitit University Erol Olçok Training and Research Hospital, Çorum Turkey

\begin{abstract}
Breast cancer is the most common cancer in women is increasing all over the world. Approximately one out of eight women are diagnosed with breast cancer, and this rate is increasing more than in previous years. The mechanism of cancer formation is highly complex and genetic changes play a key role in cancer growth and development. Oncogenes are effective on cancer formation and development, while tumor supressor genes work in the opposite direction. The winner of the war between the oncogene and the tumor supressor genes determines the outcome of the disease. The effects of these alterations are complex due to the high number of changes in a typical case of breast cancer and the interactions of the biological pathways involved. This review focuses on the more common abnormalities in oncogenes and tumor suppressor genes in human breast cancer. Understanding the role of oncogenes and tumor supresor genes on breast cancer can offer different therapeutic approaches. The detection of oncogenes leads to the prognosis of the disease and follow-up of treatment. The availability of antibodies, small synthetic molecules, cyotokines, gene therapy techniques in breast cancer, and even natural compounds that are screened for specific biological properties has greatly increased the number of candidate drugs. Early diagnosis is important and lifesaving, even though many different therapeutic approaches have been developed to treat breast cancer. The aim of our study was to examined in detail the role of oncogenes and tumor suppressor genes on breast cancer.
\end{abstract}

Keywords: Breast Cancer, Oncogene, Tumor supressor gene

\section{INTRODUCTION}

Invasive breast cancer is the most common malignant tumor for woman in the world $(1,2)$. Approximately $23 \%$ of cancers in women worldwide are breast cancer. It is about twice as common as other cancers. The incidence of breast cancer increases rapidly with age like the most other epithelial tumors. The incidence of breast cancer varies across the world. Ethnic and geographical populations show significant differences in mutation spectra and prevalence (3). For the etiology of this disease environmental factors, also diet, reproductive factors, and hormones have prominent role for the origin of breast cancer. A woman's reproductive cycle is highly important for the development of breast cancer. Breast cancer is more common in women who often experience menarche at an early age. Furthermore the disease is more common in women, who often do their first birth at a late age or nulliparous. Infertility, lack of breastfeeding, delayed menopause also increases the risk. First doctor who reported cases of breast cancer in four generations of his wife's family in 1866, is French neurologist Paul Brocaten. Ancient Greeks were first researchers who interested in familial clustering of breast cancer, but this report still remains the first well-documented about inherited breast cancer. The importance of family history was not care until the 1960s (2). More than most other human neoplasms, breast cancer shows familial genetic transition (3). Approximately 5$10 \%$ of all breast cancers are familial (4). If there is woman's mother or sister's breast cancer history, the risk of development breast cancer is much more than the other women (2).

In this review, we aimed to evaluate in detail the oncogen and tumor suppressor genes involved in the pathogenesis of breast cancer.

\section{BRCA 1 AND BRCA 2}

The genes most frequently involved in familial breast cancers are BRCA 1 and BRCA $2(1,3)$. 
The effect of mutations in specific genes such as BRCA1 and BRCA2 on prognosis is the subject of active research (5). Only an estimated 20$25 \%$ of familial breast cancer cases are explained by BRCA1/2 pathogenic variants. More than $>60 \%$ of the hereditary predisposition remains unexplained (3). Distinguishing familial cases morphologic features have been described for those breast cancers that arise in $\mathrm{CDH} 1$ and BRCA1 and possibly BRCA2 germ line mutation carriers only (6).

BRCA1 and BRCA2 are involved in DNA repair pathways and are also known to be tumor suppressor genes. Cells lacking functional BRCA1 or BRCA2 are insufficient for double-stranded fracture repair, leading to genomic imbalance and subsequent cancer susceptibility. (7).

BRCA-associated breast carcinomas have a poorly differentiated histologic grade from nonBRCA-related carcinomas. On the other hand BRCA1-associated breast tumors are usually negative for Estrogen receptor (ER), Progesterone receptor (PR) and HER2 (c-erbB$2)$; these cancers are described as "Triple negative breast cancer (TNBC)" and show a high rate of ki-67 marking signs (5). Also they are associated with Tumor protein p53 (TP53) mutation (1).

BRCA 1 is located on chromosome 17q21. It was discovered in 1990, and was identificatied BRCA1 gene sequence a few years later (8). BRCA 1-encoded protein has many roles like, chromatin remodeling and DNA decatenation, cell cycle checkpoint control, homologous recombination DNA repair, ubiquitylation, chromatin remodeling and DNA decatenation $(1,6)$. It also encodes a protein residue of 1863 amino acids in length (6). The BRCA1 protein has multiple sequence motifs including RING, DNA-binding, and BRCA1 C-terminus domains that allow BRCA1 to interact with other proteins and assist in subcellular localization (7).

BRCA1 is a tumor suppressor gene that contributes to repair of damaged replication forks and double-strand breaks, transcriptional regulation in response to DNA damage, chromatin remodeling, and regulation of cell division, apoptosis, and transcription (7).

Breast carcinomas with BRCA1 mutations tend to be highly grade, they are mitotically very active. 5 to $19 \%$ of BRCA1-associated tumors have medullary features. These tumors have insidious growth patterns, forcing margins, associated necrosis and marked nuclear pleomorphism $(1,6)$.
In terms of clinical features most BRCA1associated breast cancers have the same radiological features like mammographic findings as seen in sporadic cancers. There is a high percentage that can appear mammographic all as a well-defined mass. Both mammographically and by ultrasound examination the tumor may mimic a benign lesion (6).

BRCA2 is a gene located on chromosome 13q12. BRCA2 has 27 exons and the BRCA2 protein interacts with RAD51 through the BRC motif. BRCA2 is also a transcriptional coregulator involved in DNA repair through homologous recombination (7).

Both BRCA 1 and BRCA 2 are required for homologous recombination repair of DNA double helix breaks. If DNA repair is not achieved, cancer develops. Loss of such function in the associated cancer is being exploited to develop novel therapies- for example, poly- ADP-ribose polymerase inhibitor (blocking repair of DNA breaks in tumor cells, which are deficient in DNA repair) (1).

BRCA1-associated carcinomas showed higher medullary carcinoma, poorly differentiated histologic grades, high mitotic rate, prominent lymphocytic infiltrates and necrosis frequency than BRCA2-related carcinomas (5).

Furthermore, BRCA2-related cancers do not have a specific morphology or phenotype and are usually positive for hormone receptors (1).

\section{Checkpoint Kinase 2}

Checkpoint kinase 2 (CHEK2) encodes a serine threonine kinase involved in DNA repair. The task of this gene is to become a cell cycle control point regulator and to work as a tumor suppressor gene. Mutations in CHEK2 are found in a variety of cancers (7).

\section{Cadherin 1}

Cadherin 1 (CDH1) gene encodes an adhesion molecule involved in maintenance of epithelial cell morphology (3). Cadherin-1 also known as CAM 120/80 or epithelial cadherin(E-cadherin) or uvomorulin is a protein that in humans is encoded by the $\mathrm{CDH} 1$ gene. $\mathrm{CDH} 1$ has also been designated as CD324 (cluster of differentiation 324). It is a tumor suppressor gene.

\section{The Phosphatidylinositol 3-Kinase}

Phosphatidylinositol-4,5-bisphosphate 3-kinase catalytic a subunit (PIK3CA) is located on chromosome $3 \mathrm{q} 26.32$, that encodes the catalytic 
subunit $\mathrm{p} 110 \alpha$ of class IA Phosphatidylinositol 3-kinase (PI3K). It is one of the recently identified tumor suppressor genes and its mutations serve a major role in downstream signaling of receptor tyrosine kinases (9).

\section{Tumor Protein 53}

Tumor protein 53 (TP53), which encodes the p53 phosphoprotein, is a tumor suppressor gene. The p53 signaling pathway is at least partially disrupted in most human cancers and TP53 mutations are the most frequent genetic changes seen in human cancers (1).

\section{Phosphatase and Tensin Homolog}

Phosphatase and tensin homolog (PTEN) gene was identificated in 1997 (2). Phosphatase and tensin homolog is a tumor suppressor gene involved in the regulation of the phosphoinositol-3-kinase and AKT signaling pathways and control of cellular proliferation and survival (7).

\section{Human mutS Homolog 2}

Human mutS homolog 2 (MSH2) tumor suppressor gene plays a role in DNA mismatch repair. Mutations in $\mathrm{MSH} 2$ may contribute to genomic instability. It cause an increased mutation rate in cancer cells (7).

In recent years more data on the molecular aberrations found in distinct subtypes of Ductal carcinoma in situ (DCIS) and invasive carcinomas of ductal. Often ER+ low-grade DCIS and invasive carcinomas are characterized by expression of hormone receptors. At the same time these cancers lack of HER2 over expression, and lack of expression of basal markers. Genetic researches are show that, these lesions have rather simple, diploid/near diploid karyotype and display as hallmark recurrent changes the concurrent presence of deletion of
$16 q(>70 \%)$ and gains of $1 \mathrm{q}(>70 \%)$ and $16 \mathrm{p}$ $(>40 \%)$. Infrequently, these three genetic aberrations are the product of the $\operatorname{del}(16)$ $\mathrm{t}(1 ; 16) / \operatorname{del}(1 ; 16)$ unbalanced chromosomal translocation, which is reported to be more often found in invasive lobular carcinomas (ILCs) and low-grade DCIS/low-grade invasive carcinomas of ductal. It is remarkable, that although loss of $16 \mathrm{q}$ is the single most frequent genetic event observed in low- and intermediate-grade DCIS, the target gene marks difficult to find. (6)

At first view, intermediate-grade (i.e., grade 2) DCIS shows same genetic features to both lowand high-grade DCIS, including gain of 1q and loss of 8p, 11q, 16q, and 17p. Available findings suggest that the most of these lesions may be the product of progression of ER+ lowgrade DCIS, whereas a smaller subgroup may represent the lower end of the spectrum of DCIS with molecular aberrations characteristic of high-grade lesions. (6)

The similarities between atypical ductal hyperplasi $(\mathrm{ADH})$ and low-grade DCIS are dramatic. Immunophenotypically, both lesions are consistently positive for ER and progesterone receptor and lack HER2 over expression and gene amplification. Although one third to one half of all ADH has been shown not to harbor genetic changes, the others display genomic aberrations that are strikingly similar to those of low-grade DCIS, including loss of $16 q$ and gain of 17p. (6)

These data show us there multistep model of breast carcinogenesis. Poorly differentiated carcinomas would consistently arise through "de-differentiation" of "better differentiated" lesions. The new genes and pathways found in recent studies show that there are many ways to progress towards breast cancer and malignancy (6). (Table 2, Table 3)

Table1. Summary of the syndromes associated with hereditary breast cancer

\begin{tabular}{|c|c|c|}
\hline Syndrome & Gene and Cytoband & Clinical Properties \\
\hline $\begin{array}{l}\text { Hereditary breast cancer and ovarian } \\
\text { cancer syndrome }\end{array}$ & BRCA1 (17q21) & $\begin{array}{lccc}* \text { Breast cancer, high risk } & (50-80 \%) \\
* \text { Ovarian cancer, high risk }(40-50 \%) & \end{array}$ \\
\hline $\begin{array}{l}\text { Hereditary breast cancer and ovarian } \\
\text { cancer syndrome }\end{array}$ & BRCA2 (13q12.3) & $\begin{array}{l}\text { *Breast cancer, high risk }(50-70 \%) \\
* \text { Ovarian cancer, intermediate risk }(10 \%) \\
\text { *Prostate cancer } \\
\text { *Pancreatic cancer }\end{array}$ \\
\hline $\begin{array}{l}\text { CHEK2 mutations (Li-Fraumeni } 2 \\
\text { syndrome) }\end{array}$ & CHEK2 (22q 12.1) & $\begin{array}{l}\text { *Breast cancer, intermediate risk } \\
\text { *Sarcomas } \\
\text { *Brain tumors }\end{array}$ \\
\hline Other Fanconi anemia genes & $\begin{array}{l}\text { PALB2/FANCN (16p12) } \\
\text { FANCA (16q24.3) } \\
\text { FANCE (6p22-p21) } \\
\text { BRIP1/FANCJ (17q22) }\end{array}$ & $\begin{array}{l}\text { *PALB2/FANCN and BRIP1/FANCJ: moderate } \\
\text { risk of breast cancer development } \\
\text { *The other FANC genes: low risk of breast } \\
\text { cancer development }\end{array}$ \\
\hline
\end{tabular}


The Roles of Oncogenes and Tumor Supressor Genes in Breast Cancer Pathogenesis

\begin{tabular}{|c|c|c|}
\hline $\begin{array}{l}\text { Familial linitis plastica type gastric } \\
\text { cancer and lobular breast carcinomas } \\
\text { syndrome }\end{array}$ & CDH1 (16q22.1) & $\begin{array}{l}\text { *Gastric cancer } \\
\text { *Lobular breast cancer }\end{array}$ \\
\hline Louis-Bar syndrome & ATM (11q22.3) & $\begin{array}{l}\text { *Lymphoma } \\
* \text { Cerebellar ataxia } \\
\text { *Immune deficiency } \\
\text { *Glioma } \\
\text { *Medulloblastoma } \\
\text { *Breast cancer } \\
\end{array}$ \\
\hline Li-Fraumeni syndrome & TP53 (17p13.1) & $\begin{array}{l}\text { *High penetrance for breast cancers at young age } \\
\text { * Risk of soft tissue sarcomas and osteosarcomas, } \\
\text { brain tumors, leukemia, and adrenocortical } \\
\text { carcinoma }\end{array}$ \\
\hline Cowden syndrome & PTEN (10q23.31) & $\begin{array}{l}\text { *Increased risk of developing neoplasms (breast } \\
\text { cancer, thyroid carcinoma, endometrial } \\
\text { carcinoma and others) } \\
\text { *Hamartomatous polyps of the gastrointestinal } \\
\text { tract } \\
\text { *Mucocutaneous lesions }\end{array}$ \\
\hline $\begin{array}{l}\text { Bannayan-Riley-Rivalcaba } \\
\text { syndrome }\end{array}$ & PTEN (10q23.31) & $\begin{array}{l}\text { *Breast cancer } \\
\text { *Meningioma } \\
\text { *Follicular cell tumors of the thyroid } \\
\end{array}$ \\
\hline Peutz-Jeghers syndrome & STK11 (19p13.3) & $\begin{array}{l}* \text { Melanocytic macules of the lips, buccal mucosa, } \\
\text { and digits } \\
* \text { Multiple gastrointestinal hamartomatous polyps } \\
* \text { Increased risk of various neoplasms (breast, } \\
\text { testis, pancreas, and cervix) }\end{array}$ \\
\hline Lynch cancer family syndrome II & 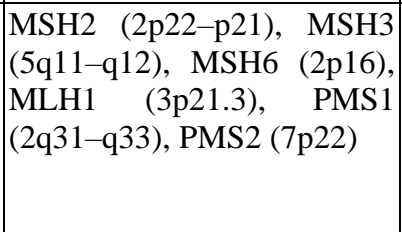 & $\begin{array}{l}\text { *Increased risk of endometrial carcinoma and } \\
\text { colorectal } \\
\text { *High risk of multiple primary malignant } \\
\text { neoplasms, including breast, ovarian, } \\
\text { gastrointestinal and genitourinary carcinomas, } \\
\text { sarcomas, glioblastoma, and leukemia }\end{array}$ \\
\hline
\end{tabular}

Table2. DCIS: Summary of morphologic, immunohistochemical, and molecular genetics features. + , Frequently positive; +/-, fairly frequent positivity; -/+, frequently negative.

\begin{tabular}{|l|c|c|c|}
\hline GRADE & $\mathbf{1}$ & $\mathbf{2}$ & $\mathbf{3}$ \\
\hline Ploidy & Near diploid & Aneuploid $(\sim 40-50 \%)$ & Frequently aneuploid \\
\hline Recurrent changes & $1 \mathrm{q}+, 16 \mathrm{q}-$ & $1 \mathrm{q}+, 8 \mathrm{p}-, 11 \mathrm{q}-, 16 \mathrm{q}-, 17 \mathrm{p}-$ & $\begin{array}{c}1 \mathrm{q}+, 3 \mathrm{q}+, 17 \mathrm{q}+, 8 \mathrm{q}+, 5 \mathrm{q}- \\
11 \mathrm{q}-, 14 \mathrm{q}-, 8 \mathrm{p}-, 13 \mathrm{q}-\end{array}$ \\
\hline Amplifications & Rare;8p11.2-p12 & $8 \mathrm{p} 11.2-\mathrm{p} 12,11 \mathrm{q} 13$ & $\begin{array}{c}17 \mathrm{q} 12,6 \mathrm{q} 22,8 \mathrm{q} 22,11 \mathrm{q} 13, \\
20 \mathrm{q} 13\end{array}$ \\
\hline Molecular subtype & Luminal A>B & Luminal A, B & Luminal B, HER2, basal \\
\hline
\end{tabular}

Table3. Low-grade ductal and lobular carcinomas have similar immunoprofiles and genetic features.

\begin{tabular}{|l|c|c|c|}
\hline & ADH & LOW- grade DCIS & ALH/LCIS \\
\hline Ploidy & diploid/near diploid & near diploid & diploid/near diploid \\
\hline Recurrent changes & $1 \mathrm{q}+, 16 \mathrm{q}-$ & $1 \mathrm{q}+, 16 \mathrm{q}-$ & $1 \mathrm{q}+, 16 \mathrm{q}-$ \\
\hline Amplifications & Rare; 8p11.2-p12 & Rare; 8p11.2-p12 & Rare; 8p11.2-p12 \\
\hline Molecular subtype & Luminal A>B & Luminal A>B & Luminal A>B \\
\hline
\end{tabular}

\section{DISCUSSION}

Breast cancer is the most common cancer in the women and its frequency is increasing $(1,2)$. Incidence varies in different regions and ethnic groups around the world. Breast cancer incidence rates are higher in populations with high socioeconomic status. Mortality and deaths are higher in populations with low socioeconomic status. There exists a variation in breast cancer incidence and mortality between different regions and countries. (10). Australia, Europe and North America are the areas of high risk for breast cancer. In these areas, invasive breast cancer develop $6 \%$ of women before age 75 years. In less developed regions of subSaharan Africa and in parts of southern and eastern Asia the risk of breast cancer is low. The probability of developing breast cancer by age 75 years is one third that of rich countries (2). There are variation in presentation, outcomes and etiologies of breast cancer. Emphasize the 
importance of further work on dissemination and prevalence (10).

As with other cancers, there are many different factors in the etiology of breast cancer. Environmental factors, diet, reproductive factor and hormones in the etiology are very important for the development of breast cancer (2).

Breast cancer shows familial genetic transition much more than the other cancers (6). There is a need to investigate and better understand the inherited risk of cancer. These studies will provide a more accurate assessment of clinical practices and management strategies (4).

Gene panels allow several genes to be screened at the same time. The panels are customized for specific scanning purposes. Thus, the genes (and / or specific gene regions) in such panels are specific for screening purposes. In recent years, with technological sequencing advances, panelbased screening has become extremely efficient and cost-effective. In recent years panel-based screening has become extremely efficient and cost-effective. These advancements involve the targeted enrichment of selected genes. Parallel sequencing, which is also known as nextgeneration sequencing gene panels have been implemented into clinical practice to assess inherited risk of cancer (4).Since the advent of high-throughput sequencing technologies, organized germ line screening is recommend to women who have family cancer history (3).

Molecular genetic analysis has change our understanding of breast lesions radically. The multistep model of breast carcinogenesis, proposing a transition from normal epithelium to invasive carcinoma via no atypical and atypical hyperplasia and in situ carcinoma, is currently overly and conceptually accepted. Over the last few years, advances in molecular pathology have begun to use molecular methods for laser capture micro dissection and formalinfixed paraffin embedded tissue samples. The morphologic and genetic features of breast cancer precursors have been viewed using loss of heterozygosis, chromosomal and microarraybased comparative genomic hybridization, fluorescent and chromogenic in situ hybridization, and microarray-based gene expression profiling (6).

Some researchers have collected genealogists with multiple breast cancer cases. These studies have shown that familial breast cancer differs in many respects from non-familial breast cancer. These differences are earlier age of onset, excess of bilaterality, certain relationships with other malignancies in the family, and vertical transitions (1).

Additional polymorphisms and genes have been recently identified primarily via genome-wide association studies which are of medium or low penetrance and convey lower risks (2).

BRCA1, the first gene identified in breast cancer genetic studies, was identified 30 years ago (8). Then other genes and mutations were detected respectively. Although the detection of these mutations has become more frequent in recent years, there are many genes and mutations that should be found. Numerous studies on these are promising.

The BRCA1 and BRCA2 genes are the best characterized breast cancer susceptibility gene. In most families with both breast and ovarian cancer or early-onset breast cancer, the disease is attributable to mutations in one of these two highly penetrate genes. Mutation carriers are also at significantly increased risk of other malignancies. The most notably cancer is ovarian carcinoma. For BRCA1 and BRCA2 mutation carriers, the risk of survival in ovarian cancer development is $44 \%$ and $27 \%$, respectively, relative to $70 \%$ (6).

In the mid-1990s, these genes are part of the DNA-repair machinery were play a important role in hereditary breast and ovarian cancer (HBOC). Pathogenic variants in these two genes explain about $24 \%$ of all HBOC cases. BRCA1 and BRCA2 are playing a substantial role in the homologous recombination repair pathway. Up to now then more than 300 gene products have been associated with the DNA-repair machinery and genome integrity maintenance, 25 out of 300 genes have been linked with HBOC. (11).

Mutations in these genes cause the development of cervix, endometrial, fallopian tube, peritoneal, gastric and liver carcinomas and prostate cancer for male carriers other than female breast and ovarian cancer (6).

Additionally BRCA1 and BRCA2, several different genes present a moderate to low risk of development of breast cancer. Hereditary breast cancer can also occur in the setting of multiple cancer syndromes, as summarized in Table 1 (1). Patients with BRCA germ line mutations had a substantial 5 year $(11.9 \%)$ and 10 year $(37.6 \%)$ risk for contralateral carcinoma and about $10 \%$ developed ovarian carcinoma (5).

In the general population the prevalence of BRCA1 mutations is approximately 1 in 1000 , 
with high penetrance. Certain ethnic or geographically distinct groups, such as those of Ashkenazi Jewish decent, French Canadians, and Finns, have a higher risk this disease because of the presence of founder mutations (6).Breast cancer associated with BRCA1 accounts for $5 \%$ of all breast cancers (8).

In female BRCA1 mutation carriers, life time risk for the development of breast cancer in mutation carriers being $37 \%$ to $85 \%$ by age 70 (3). On the other hand the risk of developing breast cancer by the age of 80 is $72 \%$. In addition, the incidence of breast cancer increases rapidly in BRCA1 mutation carriers up to 30 to 40 years of age in early adulthood (11).

The functions of this protein are numerous. First, the BRCA1 locus was thought to be limited to families with early-onset breast cancer cases (i.e. at $<50$ years). Also these studies show that the mutations in BRCA1 predispose strongly to the development of ovarian cancer. The breast- ovarian cancer syndrome had already been suggested by Henry Lynch in 1978, and was given a formal genetic basis (1).

BRCA2 is a tumor suppressor gene located on chromosome 13q12 that was identified in 1995. It was discovered by Michael Stratton and the other investigators who study with him.

Robson et al. found no significant differences in the expression of epidermal growth factor receptor, cathepsin D, bcl-2, p27, p53, or cyclin$\mathrm{D}$, and there was not a significant difference in relapse-free survival or overall survival between BRCA-associated and non-BRCA-associated tumors, despite the relatively unfavorable tumor characteristics of the former group. Other investigators have found an increased frequency of p53 mutations and lower frequency of HER2/neu amplification in BRCA1 carcinomas than in sporadic breast carcinomas (5).

In one study, $70 \%$ of BRCA1 carriers and $40 \%$ BRCA2 carriers were found in Australian women with breast cancer before the age of 40 in a first- or second-degree relative who did not have a family history of breast cancer. (5).

An increased risk of contra lateral breast carcinoma has also been found in women with familial breast cancer without a BRCA1 or BRCA2 mutation (5).

A large study evaluating CHEK2 mutations in breast cancer patients in Poland has been conducted. They found that $\mathrm{CHEK} 2$ carriers were significantly higher than ER + than ERtumors. In a similar study, it was $1.3 \%$ in hereditary TNBC patients with CHEK2 mutations in Polish women, and $11.3 \%$ in hereditary non-TNBC patients. The prevalence of pathogenic $\mathrm{CHEK} 2$ mutations was $1.6 \%$ in women with TNBC and $14.3 \%$ in other phenotypes in 35.409 women undergoing panel testing (7)

Due to its mutations in $\mathrm{CDH} 1$, there is an increased risk of breast cancer, particularly invasive lobular carcinoma. It is also associated with cancer susceptibility syndrome and has an increased risk of hereditary widespread gastric cancer. Given that invasive lobular carcinomas are often $\mathrm{ER}+$, there is no possibility of a relationship between germ line mutations in CDH1 and TNBC. Accordingly, germ line mutations in $\mathrm{CDH} 1$ were rare $(0.0-0.3 \%)$ in women with TNBC (7). Among the rare nonBRCA variants, most sequence changes affected the ATM and NF1 genes followed by the CDH1 gene. The majority of variants were missense followed by synonymous , noncoding, nonsense, and frame shift sequence variations (3).

PI3K pathway is one of the most altered ways in luminal breast cancer. Major efforts to resolve endocrine resistance indicated abnormal signaling of PI3K e.g., by PIK3CA mutations, loss of PTEN, or downstream protein phosphorylation (12).

Some studies showed that almost $21-34 \%$ of breast cancer cases exist PIK3CA mutations. PIK3CA mutations were associated with ER + /PR + tumor group as opposed to ER- /PRgroup $(\mathrm{P}=0.021)$ (9).

Last studies led to the development of treatment strategies like mTOR and PI3K inhibitors. Adding PI3K inhibitors to standard endocrine treatment results in improvement of progression free survival in patients with metastatic disease with a PIK3CA-mutated tumor. So far, it is largely unknown whether PI3K alterations influence the composition of the tumor microenvironment (12). There are some studies aimed their role in breast cancer disease progression. The role was associated with a poor disease prognosis (9).

It is emphasized the potential of PIK3CA mutations as an important biomarker for breast cancer classification and it is the possible use of PIK3CA inhibitor as targeted therapy for breast cancer. Also PIK3CA mutated oncogenes are showed at some of breast, colon and endometrial cancer. (9). 
TP53 plays a critical role in familial breast cancer. Although the frequency of somatic mutations in TP53 is higher in basal-like tumors than any other subtype, germ line mutations in TP53 have not been associated with an increased risk of TNBC (13).

Slavin et al, found the mutation rate for TP53 in a cohort of 2,134 BRCA1/BRCA2 mutation negative women with familial breast cancer was $0.52 \%$ and TP53 mutations carriers showed enrichment for HER2+ tumors (13). In Buys's study a large cohort of 35,409 women with a single diagnosis of breast cancer, mutations in TP53 were detected in $0.7 \%$ of women with TNBC compared to $2.1 \%$ of those with nonTNBC subtypes (14). In 133 women from Taiwan with early-onset and/or family history of breast cancer, only two women carried a pathogenic mutation in TP53 and both had ER+/HER2+ tumors. Similarly, only one of 1,824 women with TNBC evaluated by Couch et al. carried a TP53 mutation and these results suggest that mutations in TP53 are not associated with increased risk of TNBC (7).

PTEN gene select a specific familial cancer phenotype, as was the case for BRCA1 and BRCA2. When some families genome examined which of them affected by Cowden syndrome, researchers found PTEN mutation. These families were also found to be more frequent in multiple hamartomatous lesions, risk of neoplasms of the thyroid, breast, and female genitourinary tract than in other individuals in the community (1).

PTEN is the second most frequently mutated gene in human cancers (after TP53) and in cancer susceptibility syndromes, germline mutations in PTEN are frequently observed. PTEN has recently been shown to protect the genome from instability by preserving its chromosomal integrity. Women with Cowden syndrome with germline mutations in PTEN have a 50\% lifetime risk of breast cancer, but there is no consistent breast cancer phenotype associated with PTEN mutations. Most PTENrelated tumors are more likely to be luminal than TNBC. Ellsworth and et al. make observations that prevalence of pathogenic mutations in PTEN did not differ significantly in women with TNBC ( $n=692)$ compared to those with non TNBC tumors $(n=2,696)$ and (2) only one deleterious mutation in 267 patients was observed support the idea that mutations in PTEN are not associated with increased risk of TNBC (7).
A relatively low mutation rate $(0.7 \%)$ was observed in MSH2 in women with TNBC compared to $1.2 \%$ in women with non-TNBC. It is associated with autosomal dominant Lynch Syndrome (7).

In recent years, with technological advances, genetic screening has become extremely efficient and cost effective. These advances include enrichment of certain genes, followed by mass parallel sequencing, also known as next-generation sequencing $(15,16)$. Nextgeneration sequencing gene panels are being used in clinical practice to detect the risk of hereditary cancer $(16,17)$. As a result of all researches, guidelines are created. In the U.S., the National Comprehensive Cancer Network provides such guidelines to maximize clinical utility (18).

The use of newly discovered genes and mutations as screening tests is controversial. The aim of the screening test is to prolong survival with early diagnosis and treatment in particularly risky groups. For example routine BRCA1 / 2 assessment for the whole or female population is not currently recommended. However, according to guidelines prepared by some groups, such as the Swiss Clinical Cancer Research Group, individuals in a particular community should be directed to genetic testing, regardless of their personal or family cancer background (19). Traditional estimates of BRCA $1 / 2$ mutation carrier prevalence, which range from $0.2 \%$ to $0.3 \%$ (1:300 to $1: 500)$ in the general female population (20). Dennis et al. found in our total cohort a significantly higher carrier rate of $0.8 \%$ (3). This shows that the mutation rate varies from community to community. The necessity of applying genetic screening test also varies among societies.

Therefore, diagnosis and treatment are of great importance. Today, only the diagnosis is inadequate. There are many subtypes of breast cancer. As the subtypes were found, advancing technologies and patient-specific treatments were also discovered. Subtypes have started to be made according to genetic mutations along with histomorphological features. Sub typing and using appropriate treatment methods are important for survival.

Also breast cancer leading cause of carcinoma death (1). In the world, there has been a sharp increase in the detection due to the widespread use of mammography (1). In increase in the number of earlier and smaller case breast cancer mortality is beginning to fall because of the 
combined earlier diagnosis and improved therapy (1). Immunohisto chemical staining and genetic studies are important in early diagnosis and treatment of the breast cancer.

Consequently some authors say that the patients should be referred for genetic testing, irrespective of their personal or family cancer history. Genetic testing is extremely important in terms of diagnosis, individualized treatment program, prognosis and follow-up of other disease-free family members.

\section{CONCLUSION}

Breast cancer, that incidence has increased over the years, is diagnosed more frequently with advancing technologies and research. Additional procedures during diagnosis are important in detecting subtypes of breast cancer. The identified subtypes lead to the development of new treatment methods. These treatment methods are patient-specific and aim to achieve the best targeted results. Each new gene and mutation found is in fact intended to find new treatment methods aimed at prolonging patient survival. Therefore, it is very important to add new ones to genetic research.

Future studies should focus on exploring the genetic profile of the female population in order to predict appropriate diagnostic methods.

\section{REFERENCES}

[1] Rosai and Ackerman's Surgical Pathology, 10. Edition, 2011, Elsevier, volume 2, chapter 20, p. 1681-1684

[2] World Health Organization Classification of Tumors, 4th Edition, 2012, Lyon, Internation Agency for Research on Cancer, Chapter 1, p.14-31, chapter 16, p. 171-195

[3] Dennisa K, Silviaa AB, Katharinaa S, Paranchaia B, Markusa Z, Konstantin D, Pascala J, Danielb F, Anita R. Prevalence of Genetic Susceptibility for Breast and Ovarian Cancer in a Non-cancer Related Study Population: Secondary Germline Findings from a Swiss Single Centre Cohort, Swiss Med Wkly. 2019;149:w20092

[4] Bishop MR, Huskey ALW, Hetzel J, MernerND. A Research-based Gene Panel to Investigate Breast, Ovarian and Prostate Cancer Genetic Risk, PLoS One, 2019 Aug 15;14(8)

[5] Rosen's Breast Pathology, 3rd Edition, Chapter 12 - Invasive Duct Carcinoma: Assessment of Prognosis, Morphologic Prognostic Markers, and Tumor Growth Rate, p. 358-404

[6] Breast Pathology, O'malley, 2 nd Edition, chapter 16, p 211-215
[7] EllsworthDL, TurnerCE, EllsworthRE.A Review of the Hereditary Component of Triple Negative Breast Cancer: High-and ModeratePenetrance Breast Cancer Genes, LowPenetrance Loci, and the Role of Nontraditional GeneticElements. J Oncol. 2019 Jul 9; 2019: 4382606

[8] Shimomura A, Yonemori K, Yoshida M, Yoshida T, Yasojima $\mathrm{H}$, Masuda $\mathrm{N}$, et al. Gene Alterations in Triple-Negative Breast Cancer Patients in a Phase I/II Study of Eribulin and Olaparib Combination Therapy. Transl Oncol, 2019 Oct;12(10):1386-1394.

[9] Vatte C, Al Amri AM, Cyrus C, Chathoth S, Alsayyah A, Ahmad A, et al. Helical and Kinase Domain Mutations of PIK3CA, and Their Association with Hormone Receptor Expression in Breast Cancer, Oncol Lett. 2019 Sep;18(3):2427-2433

[10] Fares MY, Salhab HA, Khachfe HH, Khachfe HM. Breast Cancer Epidemiology Among Lebanese Women: An 11-Year Analysis. Medicina (Kaunas), 2019 Aug 10;55(8)

[11] Sepahi I, Faust U, Sturm M, Bosse K, Kehrer M, Heinrich T, et al. Investigating the effects of additional truncating variants in DNA repair genes on breast cancer risk in BRCA1 positive women, BMC Cancer, 2019, 19:787

[12] Sobral-Leite, Salomon I, Opdam M, Kruger DT, Beelen KJ, Noort V, et al. Cancer-immune Interactions in ER-positive Breast Cancers: PI3K Pathway Alterations and Tumorinfiltrating Lymphocytes, Breast Cancer Research, 2019, 21:90

[13] Slavin TP, Maxwell KN, Lilyquist J, et al., "Te contribution of pathogenic variants in breast cancer susceptibility genes to familial breast cancer risk," Npj Breast Cancer, vol. 3, no. 1, 2017

[14] Buys SS, Sandbach JF, Gammon A, et al., "A study of over 35,000 women with breast cancer tested with a 25-gene panel of hereditary cancer genes," Cancer, vol. 123, no. 10, pp. 17211730, 2017

[15] Easton DF, Pharoah PD, Antoniou AC, Tischkowitz M, Tavtigian SV, Nathanson KL, et al. Gene-panel sequencing and the prediction of breast-cancer risk. N Engl J Med. 2015; 372(23):2243-57

[16] Shah PD, Nathanson KL. Application of PanelBased Tests for Inherited Risk of Cancer. Annu Rev Genomics Hum Genet. 2017; 18:201-27

[17] Spurdle AB, Bowman MA, Shamsani J, Kirk J. Endometrial cancer gene panels: clinical diagnostic vs research germline DNA testing. Mod Pathol. 2017; 30(8):1048-68.

[18] National Comprehensive Cancer Network. NCCN Guidelines \& Clinical Resources 2018 
The Roles of Oncogenes and Tumor Supressor Genes in Breast Cancer Pathogenesis

[Available from: https://www.nccn. org/ professionals/physician_gls/default.aspxdetection.

[19] Chappuis POBB, Bürki N, Buser K, Heinimann $\mathrm{K}$, Monnerat $\mathrm{C}$, et al. Swiss guidelines for counselling and testing - Genetic predisposition to breast and ovarian cancer. Schweiz Arzteztg. 2017;98(2122):682-4
[20] Nelson HD, Pappas M, Zakher B, Mitchell JP, Okinaka-Hu L, Fu R. Risk assessment, genetic counseling, and genetic testing for BRCArelated cancer in women: a systematic review to update the U.S. Preven tive Services Task Force recommendation. Ann Intern Med. 2014; 160(4): 255-66

Citation: Havva Hande Keser Şahin \& Mustafa Şahin, "The Roles of Oncogenes and Tumor Supressor Genes in Breast Cancer Pathogenesis”, International Journal of Research Studies in Medical and Health Sciences. 2020; 5(5): 06-14.

Copyright: (c) 2020 Havva Hande Keser Şahin \& Mustafa Şahin, This is an open-access article distributed under the terms of the Creative Commons Attribution License, which permits unrestricted use, distribution, and reproduction in any medium, provided the original author and source are credited. 